\title{
Elevation of blood $\beta$-carboline alkaloids in essential tremor
}

\author{
E.D. Louis, MD, MS; W. Zheng, PhD; E.C. Jurewicz, BA; D. Watner, MA; J. Chen, PhD; \\ P. Factor-Litvak, $\mathrm{PhD}$; and M. Parides, $\mathrm{PhD}$
}

\begin{abstract}
Background: $\beta$-Carboline alkaloids are normal body constituents but are also potent tremor-producing chemicals that are naturally present in the food chain. Objective: To explore the hypothesis that high concentrations of $\beta$-carboline alkaloids are associated with essential tremor (ET). Methods: One hundred cases and 100 controls were frequency matched on age, sex, and ethnicity. Blood concentrations of harmane and harmine were quantified by highperformance liquid chromatography, blinded to clinical information. Results: The mean log blood concentration of harmane was higher in cases than controls $\left(0.72 \pm 0.53 \mathrm{vs} 0.51 \pm 0.64 \mathrm{~g}^{-10} / \mathrm{mL} ; p=0.01\right)$. A nonparametric test on nontransformed data (median harmane $=5.21 \mathrm{~g}^{-10} / \mathrm{mL}$ in cases and $2.28 \mathrm{~g}^{-10} / \mathrm{mL}$ in controls) confirmed this difference $(p=0.005)$. The mean log blood concentration of harmine was $0.20 \pm 0.48 \mathrm{~g}^{-10} / \mathrm{mL}$ in cases and $0.10 \pm 0.65 \mathrm{~g}-10 / \mathrm{mL}$ in controls $(p=$ 0.20). Log harmane concentrations were stratified based on the median value; $62 \%$ of cases vs $39 \%$ of controls had a high $\log$ harmane concentration $(p=0.001)$. Mean log harmane concentration was similar in the cases with $(0.74 \pm 0.58$ $\left.\mathrm{g}^{-10} / \mathrm{mL}\right)$ and without $\left(0.71 \pm 0.50 \mathrm{~g}^{-10} / \mathrm{mL}\right)$ an affected relative $(p=0.83)$. Conclusions: Blood concentrations of harmane were measured in ET cases compared with controls. Concentrations were elevated in cases with and without a family history of ET.
\end{abstract}

NEUROLOGY 2002;59:1940-1944

Essential tremor (ET) affects 1 to $6 \%$ of the population. ${ }^{1,2}$ The pathogenesis is poorly understood, but there may be cell damage or death in the cerebellum. ${ }^{3}$ Although genetic susceptibility is important, ${ }^{4}$ nongenetic factors may contribute to the etiology $y^{5}$; specific environmental factors, however, have not been identified.

The $\beta$-carboline alkaloids, including harmine and harmane, are a group of highly tremorogenic chemicals. Laboratory animals injected with large doses of these chemicals acutely exhibit an action tremor that resembles ET. ${ }^{6}$ Human volunteers acutely exposed to large doses display a reversible coarse tremor. ${ }^{7,8}$ The effect of low-level, chronic exposure to $\beta$-carboline alkaloids is unknown.

Sources both exogenous and endogenous to the body exist. $\beta$-Carboline alkaloids are naturally present in small concentrations in the food chain, in commonly eaten plant-derived foods (wheat, rice, corn, barley), beverages (wine, beer, whisky, and sake), and inhaled substances (tobacco), ${ }^{9,10}$ although their concentrations have been quantified in few food items. Also, these chemicals are normal body constituents and are produced in vivo by the cyclization of indole-alkylamines with aldehydes, occurring in most tissues in animals and humans. ${ }^{11,12}$ Under physiologic conditions, the magnitude of exogenous vs endogenous sources has not been studied. Many $\beta$-carboline alkaloids are highly lipophilic, ${ }^{13,14}$ and brain concentrations in rats are as much as 55 times higher than those of the plasma. ${ }^{11}$ The specificity of individual $\beta$-carboline alkaloids for different brain receptors suggests that they probably have normal biological functions. ${ }^{12}$ To explore the hypothesis that high concentrations of $\beta$-carboline alkaloids are associated with ET, blood concentrations of harmane and harmine, which are among the most tremorogenic and most well-studied $\beta$-carbolines, were measured in ET cases and control subjects.

Methods. Participants. Recruitment began in 1999. The 100 ET cases were initially ascertained from a community survey and then from a tertiary referral center. As described previously, ${ }^{15}$ community cases had been participants in a population-based familial aggregation study of ET in the Washington Heights-Inwood community in northern Manhattan, NY (1995 to 1999). Seventeen were alive and had contact information when recruitment began for the current study. These were frequency matched by 5-year age strata, sex, and ethnicity (white, African American, Hispanic) to 17 control subjects from the same community-based study. An additional 83 ET cases were recruited between 2000 and 2002. All 83 were patients seen at the Neurologic Institute of New York, ColumbiaPresbyterian Medical Center. They were identified from a

From the Gertrude H. Sergievsky Center (Dr. Louis, E.C. Jurewicz and D. Watner) and Departments of Neurology (Dr. Louis) and Pharmacology (Dr. Zheng),

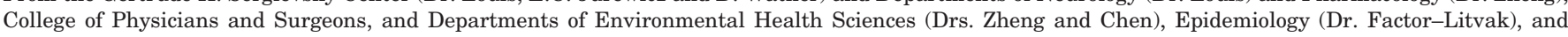
Biostatistics (Dr. Parides), Mailman School of Public Health, Columbia University, New York, NY.

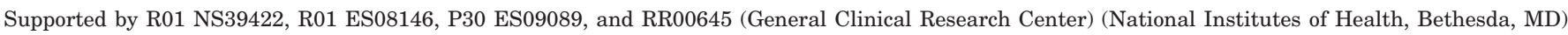
and the Paul Beeson Physician Faculty Scholars in Aging Research Award (American Federation for Aging Research, New York, NY).

Received May 23, 2002. Accepted in final form August 23, 2002.

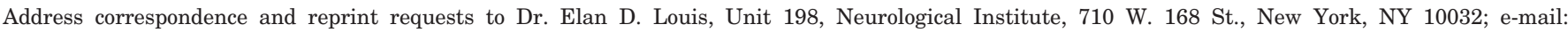
EDL2@columbia.edu

1940 Copyright (ㄷ 2002 by AAN Enterprises, Inc 
computerized database listing names and diagnoses of all patients billed within the last 3 years supplemented by a computerized database at the Center for Parkinson's Disease and Other Movement Disorders, Columbia-Presbyterian Medical Center, which listed names and diagnoses of patients seen within the last 10 years. All patients had received a diagnosis of ET from their treating neurologist at the institute. ET patients were selected, alphabetically, for enrollment. Office records were reviewed, and patients with diagnoses or physical signs of dystonia, PD, or spinocerebellar ataxia were excluded. From 2000 to 2002, 83 control cases were identified from the New York metropolitan area using random digit dialing. These control cases were frequency matched to Columbia-Presbyterian Medical Center cases on 5-year age strata, sex, and ethnicity.

Clinical evaluation. A trained tester evaluated all subjects in person. The tester was trained for 1 month by a neurologist to administer clinical questionnaires and to perform a videotaped examination. Most evaluations were home visits and therefore were performed in the late morning or early afternoon, making fasting levels impractical. Data suggest that plasma concentrations of harmane do not change significantly after meals. ${ }^{16}$ In one study, ${ }^{16}$ human subjects ingested food or ethanol, and plasma harmane concentrations were measured hourly for 8 hours. The concentration remained stable. The same investigators also demonstrated that variability in concentration was minimal over a longer (3-week) period. ${ }^{16}$

The tester collected demographic, clinical, and family history information. Data on current diet were collected using a Willett Semi-Quantitative Food-Frequency Questionnaire. This 20-minute food-frequency questionnaire ${ }^{17}$ included questions on frequency of current consumption of 61 foods and on the use of vitamins and mineral supplements. Food frequency data may be used to compute mean daily caloric, protein, carbohydrate, fat, and ethanol intake. The questionnaire has shown good reliability and validity related to recent nutrient intake. ${ }^{17}$ Subjects were considered current ethanol users if they drank at least one alcoholic beverage (beer, wine, other) per month. Current smoking status was assessed in each subject. Medical comorbidity was assessed with the Cumulative Illness Rating Scale, in which the subject reported the presence or absence of illness within 14 bodily systems (cardiac; vascular; respiratory; ear, nose and throat; upper gastrointestinal; lower gastrointestinal; hepatic; renal; genitourinary; musculoskeletal; hypertension; neurologic [other than ET]; endocrine; malignancy). ${ }^{18}$ The names of all current medications were recorded separately and then also as 19 classes of medications (e.g., neuroleptic medications, cardiac medications, anticancer medications, narcotics, medications for ET, selective serotonin reuptake inhibitors, oral hypoglycemic agents).

Weight and height were assessed using a balance scale designed for field surveys (Scale-Tronix 5600; White Plains, NY) and a movable anthropometer (GPM Martin Type; Pfister, Carlstadt, NJ). Body mass index was calculated as weight in kilograms divided by the square of height in meters.

The tester videotaped a tremor examination in all 200 subjects, ${ }^{15,19}$ and each of 12 videotaped action tremor items was rated by the neurologist on a scale from 0 to 3 , resulting in a total tremor score (range $=0$ to 36 [maximum]).
The neurologist also noted the presence or absence of head tremor on the videotaped examination. The diagnosis of ET was confirmed by the neurologist using published diagnostic criteria (moderate amplitude tremor during three activities or a head tremor). ${ }^{15,19}$ None of the cases or control subjects had PD or dystonia.

Blood harmane and harmine concentrations. During the clinical evaluation, phlebotomy was performed. Blood concentrations of harmane and harmine were measured, blinded to any clinical information including age, sex, and diagnosis. Samples were run in batches of 10 , containing an approximately equal number (4 to 6 ) of cases and control specimens. A novel high-performance liquid chromatography (HPLC) method for quantifying harmane and harmine in blood has been reported previously. ${ }^{20}$ One volume $(9$ to $12 \mathrm{~mL}$ ) of whole blood was mixed with a halfvolume (5 to $6 \mathrm{~mL}$ ) of $1 \mathrm{~mol} / \mathrm{L} \mathrm{NaOH}$. Following vortexing for 30 seconds, the samples were placed on a horizontal rotator and shaken at room temperature for 30 minutes. An aliquot $(15 \mathrm{~mL})$ of the extraction solution consisting of ethyl acetate and methyl-t-butyl ether $(2: 98, \mathrm{vol} / \mathrm{vol})$ was added to the tube. The tube was then vigorously shaken by hand for 1 to 2 minutes, followed by shaking on a horizontal rotor at room temperature for 45 minutes. After centrifugation at $3,000 \mathrm{~g}$ for 10 minutes, the upper organic phase was separated. The extraction procedure was repeated two additional times. The organic phase was combined and evaporated under nitrogen to dryness. The samples were reconstructed in $0.25 \mathrm{~mL}$ of methanol. After centrifugation at 3,000 $\mathrm{g}$ for 10 minutes, the supernatant was transferred to autosampler vials with sealed caps for HPLC analysis.

A Perkin-Elmer model LC-250 binary liquid chromatographic system (Foster City, CA) equipped with an LC-600 autosampler and an LS-40 fluorescence detector was used for separation and quantitation. Separation was accomplished using an ion-interaction, reversed-phase Econosphere $\mathrm{C}_{18}$ column (ODS2; $5 \mu \mathrm{m}, 250 \times 4.6 \mathrm{~mm}$ ) attached to a Spherisorb guard column (ODS2; $5 \mu \mathrm{m}, 10 \times 4.6 \mathrm{~mm}$ ). Both analytical and guard columns were purchased from Alltech (Deerfield, IL). An isocratic mobile phase consisted of $17.5 \mathrm{mmol} / \mathrm{L}$ of potassium phosphate buffer $(\mathrm{pH} 6.5$; equal molar concentration of both monobasic and dibasic potassium salts) and methanol (30:70, vol/vol). A $50-\mu \mathrm{L}$ aliquot of sample extracts was injected and the separation performed at room temperature at a flow rate of $1 \mathrm{~mL} / \mathrm{min}$. The detector was set at an excitation wavelength of 300 $\mathrm{nm}$ and an emission wavelength of $435 \mathrm{~nm}$. A Macintosh computer equipped with Mac Integrator II (Rainin; Apple, New York, NY) was used to collect and analyze the data. The identity of harmane and harmine on high-performance liquid chromatographs previously has been clarified. ${ }^{14,20}$ The intraday precision, measured as a coefficient of variation at $25 \mathrm{ng} / \mathrm{mL}$, was $6.7 \%$ for harmane and $3.4 \%$ for harmine. The interday precision was $7.3 \%$ for harmane and $5.4 \%$ for harmine. ${ }^{20}$

Statistical analyses. The empirical distributions of both harmane and harmine were positively skewed. Using a onesample Kolmogorov-Smirnov test, we tested whether harmane and harmine concentrations were normally distributed. Rejection of the null hypothesis (i.e., $p<0.05$ ) is consistent with a nonnormal distribution. Neither harmane nor harmine was normally distributed (Kolmogorov-Smirnov test, $z=4.23$ and 5.31, both $p<0.001$ ). Therefore, harmane 
and harmine concentrations were logarithmically transformed and case-control differences compared using Student's $t$-tests. In confirmatory analyses, a nonparametric (Mann-Whitney $U$ ) test also was performed on harmane and harmine data that were not logarithmically transformed. In some analyses, log blood harmane and log blood harmine concentrations were stratified, based on the median values for the entire sample $(n=200)$, into those with high vs low values. Also, concentration quartiles were determined. $\chi^{2}$ tests were used to analyze proportions and Student's $t$-test to examine group differences in continuous variables. Pearson $(r)$ correlation coefficients were used to assess correlations between continuous variables. When comparing the proportion of cases vs controls who reported illness in each of the 14 bodily systems and when comparing the proportion of cases and controls who were taking a particular class of medication, Bonferroni corrections were used. To assess the null hypothesis that blood harmane concentration was not a predictor of diagnostic group (ET vs control), data were assessed using a logistic regression analysis with diagnostic group as the outcome and blood harmane concentration and age as independent variables. This resulted in odds ratios $(\mathrm{OR})$ with 95\% CI. Statistical analyses were performed in SPSS (version 9.0; Chicago, IL).

Our sample size calculation necessitated 100 cases and 100 control subjects. This sample size would provide 80\% power to detect a difference between cases and controls such that $60 \%$ of cases vs $40 \%$ of controls would have blood harmane or harmine concentrations above the median values for these chemicals.

Results. There were $100 \mathrm{ET}$ cases and 100 controls (mean age $=68.4 \pm 15.4$ vs $64.7 \pm 13.5$ years; $t=1.80, p=0.07$ ). Sex was identical (57\% women in each group) and ethnicity nearly identical ( 80 vs $81 \%$ white; $\chi^{2}=0.03, p=0.86$ ). Three cases and two controls were current cigarette smokers. Mean \pm SD pack-years did not differ between these three cases and two controls $(10.3 \pm 9.5$ vs $15.0 \pm 7.1$ pack-years; $t=0.58$, $p=0.60$ ). Cases and controls did not differ in terms of the proportion who reported illness in each of the 14 bodily systems on the Cumulative Illness Rating Scale or, with the exception of medications for ET, in terms of the proportion who reported currently taking each of the different classes of medication. Cases had a higher mean total tremor score than controls $(18.9 \pm 8.1$ vs $4.6 \pm 3.0 ; t=16.6, p<0.001)$. Mean disease duration (cases) was $21.3 \pm 19.3$ years. Thirty-four cases were taking a medication to treat ET.

Using our control sample, we examined potential confounding variables. Age was marginally correlated with log blood harmane $(r=0.18, p=0.08)$ and log blood harmine $(r=0.16, p=0.11)$ concentrations. There was no difference between sexes in mean log blood harmane concentration. Mean log blood harmine concentration was $-0.04 \pm 0.61$ $\mathrm{g}^{-10} / \mathrm{mL}$ in men and $0.21 \pm 0.66 \mathrm{~g}^{-10} / \mathrm{mL}$ in women $(t=1.92, p=0.06)$. There were no ethnic differences in mean log blood harmane (for whites $=0.53 \pm 0.65 \mathrm{~g}^{-10} / \mathrm{mL}$ vs nonwhites $\left.=0.43 \pm 0.64 \mathrm{~g}^{-10} / \mathrm{mL} ; t=0.58, p=0.56\right)$ or mean log blood harmine (for whites $=0.08 \pm 0.63 \mathrm{~g}^{-10} / \mathrm{mL}$ vs nonwhites $=0.17 \pm 0.73 \mathrm{~g}^{-10} / \mathrm{mL} ; t=0.50, p=0.62$ ) concentrations. There was no correlation between body mass index and log blood harmane or log blood harmine concentrations. In separate analyses for cases and controls, the mean log blood harmine and mean log blood harmane concentrations did not differ between current ethanol users and nonusers.
Table 1 Log blood harmane concentration by quartile

\begin{tabular}{lcc}
\hline Quartile & ET cases, $\mathrm{n}=100$ & Controls, $\mathrm{n}=100$ \\
\hline 1 (lowest) & 23 & 32 \\
2 & 15 & 29 \\
3 & 34 & 17 \\
4 (highest) & 28 & 22 \\
\hline
\end{tabular}

$\chi^{2}=12.31, p=0.006$.

$\mathrm{ET}=$ essential tremor.

Log blood harmane concentration was correlated with $\log$ blood harmine concentration $(r=0.28, p<0.001)$. The mean log blood harmane concentration was higher in cases than in controls $\left(0.72 \pm 0.53\right.$ vs $0.51 \pm 0.64 \mathrm{~g}^{-10} / \mathrm{mL} ; t=$ $2.56, p=0.01$ ). A nonparametric test (Mann-Whitney $U$ ) on nontransformed data (median harmane $=5.21 \mathrm{~g}^{-10} / \mathrm{mL}$ in cases and $2.28 \mathrm{~g}^{-10} / \mathrm{mL}$ in controls) confirmed this difference $(z=2.78, p=0.005)$. There was no difference in mean log blood harmane concentration in the cases who were taking a medication to treat ET vs those who were not $\left(0.76 \pm 0.59\right.$ vs $0.70 \pm 0.51 \mathrm{~g}^{-10} / \mathrm{mL} ; t=0.52, p=$ $0.60)$.

The mean log blood harmine concentration was $0.20 \pm$ $0.48 \mathrm{~g}^{-10} / \mathrm{mL}$ in cases and $0.10 \pm 0.65 \mathrm{~g}^{-10} / \mathrm{mL}$ in controls $(t=1.29, p=0.20)$. A nonparametric test (MannWhitney $U$ ) on nontransformed data (median harmine = $1.60 \mathrm{~g}^{-10} / \mathrm{mL}$ in cases and $0.65 \mathrm{~g}^{-10} / \mathrm{mL}$ in controls) provided similar results $(z=1.32, p=0.19)$. Mean log blood harmine concentration did not differ in the cases who were taking a medication to treat ET vs those who were not $\left(0.09 \pm 0.55\right.$ vs $\left.0.25 \pm 0.44 \mathrm{~g}^{-10} / \mathrm{mL} ; t=1.56, p=0.12\right)$.

Strata were created based on the median log blood harmane $\left(0.59 \mathrm{~g}^{-10} / \mathrm{mL}\right)$ and median log blood harmine $(0.18$ $\mathrm{g}^{-10} / \mathrm{mL}$ ) concentrations. Sixty-two percent of cases vs $39 \%$ of controls had a high log blood harmane concentration $\left(\chi^{2}=10.58, p=0.001\right)$, and $56 \%$ of cases vs $44 \%$ of controls had a high log blood harmine concentration $\left(\chi^{2}=\right.$ 2.88, $p=0.09$ ). The log blood harmane and log blood harmine concentrations were further divided into quartiles. The proportion in each log blood harmane quartile differed in cases and controls $\left(\chi^{2}=12.31, p=0.006\right.$; table 1 ). The proportion in each log blood harmine quartile did not differ $\left(\chi^{2}=4.67, p=0.20\right.$; table 2$)$.

In a logistic regression analysis, log blood harmane concentration $(\mathrm{OR}=1.80,95 \% \mathrm{CI}=1.10$ to $2.93, p=0.02)$ was associated with the outcome (diagnosis of ET vs normal), but age was not ( $\mathrm{OR}=1.02,95 \% \mathrm{CI}=1.00$ to $1.04, p=0.12$ ). In a logistic regression analysis, log blood harmane quartile $(\mathrm{OR}=1.32,95 \% \mathrm{CI}=1.02$ to 1.69 ,

Table 2 Log blood harmine concentration by quartile

\begin{tabular}{lcc}
\hline Quartile & ET cases, $\mathrm{n}=100$ & Controls, $\mathrm{n}=100$ \\
\hline 1 (lowest) & 12 & 21 \\
2 & 32 & 35 \\
3 & 30 & 20 \\
4 (highest) & 26 & 24 \\
\hline
\end{tabular}

$\chi^{2}=4.67, p=0.20$.

$\mathrm{ET}=$ essential tremor. 
$p=0.03$ ) was associated with the outcome (diagnosis), but age was not $(\mathrm{OR}=1.02,95 \% \mathrm{CI}=1.00$ to $1.04, p=0.13)$.

Among ET cases, the correlation $(r)$ between log blood harmane concentration and total tremor score was 0.16 $(p=0.10)$. There was no correlation between total tremor score and log blood harmine concentration $(r=-0.08, p=$ 0.43). Disease duration was not correlated with either log blood harmane or log blood harmine concentration $(r=0.03$, $p=0.76$, and $r=-0.13, p=0.24)$. We stratified the ET cases by family history, and there was no difference in mean log blood harmane concentration in those with $(\mathrm{n}=42$; $\left.0.74 \pm 0.58 \mathrm{~g}^{-10} / \mathrm{mL}\right)$ vs those without $(\mathrm{n}=58 ; 0.71 \pm 0.50$ $\mathrm{g}^{-10} / \mathrm{mL} ; t=0.22, p=0.83$ ) an affected relative. Results were similar for mean log blood harmine concentration $\left(0.16 \pm 0.54\right.$ vs $\left.0.23 \pm 0.44 \mathrm{~g}^{-10} / \mathrm{mL} ; t=0.72, p=0.47\right)$.

There were no differences between cases and controls in reported mean daily calories $(1,444.0 \pm 418.9$ vs $1420.4 \pm$ $523.3 \mathrm{kcal} ; t=0.28, p=0.78$ ) or mean daily grams of protein $(67.1 \pm 23.2$ vs $64.0 \pm 20.2 \mathrm{~g} ; t=0.83, p=0.41)$, carbohydrate $(182.0 \pm 66.2$ vs $178.2 \pm 85.8 \mathrm{~g} ; t=0.28 p=$ $0.78)$, fat $(27.1 \pm 11.6$ vs $27.2 \pm 13.8 \mathrm{~g} ; t=0.02, p=0.98)$, or ethanol $(8.1 \pm 12.9$ vs $7.4 \pm 10.1 \mathrm{~g} ; t=0.35, p=0.72)$.

Discussion. Low doses of $\beta$-carboline alkaloids are present in food (e.g., $2 \times 10^{-3} \mathrm{mg}$ of harmane in one 8 -ounce glass of wine). ${ }^{9,10} \beta$-Carboline alkaloids are normal body constituents as well. ${ }^{11,12}$

We demonstrated an increased blood concentration of harmane in ET cases. Concentrations of harmine and harmane were correlated. Harmane may be metabolized to form harmine. ${ }^{14}$ Although concentrations of harmine were elevated in cases compared with controls, the difference was not significant. This consistency of direction, but difference in strength of association, suggests that the same process may have been operating more strongly for harmane than for harmine.

The normal physiologic level of harmane in whole blood or the relationship in humans between whole blood, urine, and CSF or CNS concentrations has not been studied. In rats given an intraperitoneal injection of norharmane, a related compound, brain concentrations were several fold higher than plasma concentrations and were proportional to the intraperitoneal dose. ${ }^{11}$ Many $\beta$-carboline alkaloids are highly lipophilic, so that third-compartment sequestration in the brain is likely to occur.

This study did not provide an explanation for the case-control difference. Possibilities include increased exogenous (dietary) exposure or increased endogenous production or impaired metabolism in ET cases. Increased dietary exposure is difficult to assess because harmane and harmine concentrations have not been quantified in most food items. In one study, plasma concentrations were minimally variable over a 3-week period, suggesting that alterations in plasma $\beta$-carboline concentrations reflect long-standing metabolic alterations (i.e., increased formation or reduced metabolism). ${ }^{16}$ Little is known about the normal endogenous production or the metabolism of these compounds in humans.
Some evidence has linked ET with an enhanced expression of brain catecholamines. In one study, ${ }^{21} \mathrm{a}$ high brain level of norepinephrine was observed in three ET cases compared with three controls. This observation is consistent with the efficacy of adrenergic $\beta$-receptor-blocking agents in treating ET. ${ }^{22}$ Whereas peripheral $\beta$-adrenergic receptors probably mediate the effects of $\beta$-adrenergic-blocking agents, central mechanisms may be involved as well. ${ }^{23,24}$ Norepinephrine is metabolized primarily by monoamine oxidase type A. ${ }^{25}$ Several of the $\beta$-carbolines have been shown to inhibit monoamine oxidase, ${ }^{26,27}$ particularly type A. ${ }^{28,29}$

We did not assess fasting levels of harmane and cannot exclude the possibility that the difference we observed may reflect a difference between cases and controls in dietary intake of harmane rather than an endogenous difference in the metabolism of harmane. Also, we did not assess liver function, variability in the cytochrome P450 system, or renal function to see whether these factors, which could influence the metabolism of harmane or harmine, differed in cases and controls.

\section{References}

1. Louis ED, Ottman R, Hauser WA. How common is the most common adult movement disorder? Estimates of the prevalence of essential tremor throughout the world. Mov Disord 1998;13:5-10.

2. Louis ED, Ford B, Frucht S, Barnes LF, Tang M-X, Ottman R. Risk of tremor and impairment from tremor in relatives of patients with essential tremor: a community-based family study. Ann Neurol 2001;49:761-769.

3. Louis ED, Shungu D, Chan S, Mao X, Jurewicz EC, Watner D. Metabolic abnormality in essential tremor: a ${ }^{1} \mathrm{H}$ magnetic resonance spectroscopic imaging study. Neurology 2002;58(suppl 3):A253.

4. Gulcher JR, Jonsson P, Kong A, et al. Mapping of a familial essential tremor gene, FET1, to chromosome 3q13. Nat Genet 1997; 17:84-87.

5. Louis ED. Etiology of essential tremor: should we be searching for environmental causes? Mov Disord 2001;16:822-829.

6. Fuentes LA, Longo VG. An investigation on the central effects of harmine, harmaline and related B-carbolines. Neuropharmacology 1991;10:15-23.

7. Lewin L. Untersuchungen uber Banisteria caapi Sp. Arch Exp Pathol Pharmacol 1928;129:133-149.

8. Pennes HH, Hoch PH. Psychotomimetics, clinical and theoretical considerations: harmine, Win-2299 and Nalline. Am J Psychiatry 1957;13:885-892.

9. Adachi J, Mizoi Y, Naito T, Yamamoto K, Fujiwara S, Ninomiya I. Determination of $\beta$-carbolines in foodstuffs by high performance liquid chromatography and high performance liquid chromatograph-mass spectrometry. J Chromatogr 1991; 538:331-339.

10. Poindexter EH, Carpenter RD. The isolation of harmane and norharmane from tobacco and cigarette smoke. Phytochemistry 1962;1:215-221.

11. Fekkes D, Bode WT. Occurrence and partition of the $\beta$-carboline norharmane in rat organs. Life Sci 1993;52:20452054.

12. Rommelspacher H, May T, Susilo R. $\beta$-Carbolines and tetrahydroisoquinolines: detection and function in mammals. Planta Med 1991;57(suppl 1):S85-S92.

13. Zetler G, Singbartl G, Schlosser L. Cerebral pharmacokinetics of tremor-producing harmala and iboga alkaloids. Pharmacology 1972;7:237-248. 
14. Guan Y, Louis ED, Zheng W. Toxicokinetics of tremorogenic natural products, harmane and harmine, in male SpragueDawley rats. J Toxicol Environ Health 2001;64:101-116.

15. Louis ED, Ottman RA, Ford B, et al. The Washington Heights Essential Tremor Study: methodologic issues in essentialtremor research. Neuroepidemiology 1997;16:124-133.

16. Rommelspacher H, Schmidt LG, May T. Plasma norharmane ( $\beta$-carboline) levels are elevated in chronic alcoholics. Alcohol Clin Exp Res 1991;15:553-559.

17. Willett WC, Sampson L, Stampfer MJ, et al. Reproducibility and validity of a semiquantitative food frequency questionnaire. Am J Epidemiol 1985;122:51-65.

18. Linn BS, Linn MW, Gurel L. Cumulative illness rating scale. J Am Geriatr Soc 1968;16:622-626.

19. Louis ED, Ford B, Lee H, Andrews H. Does a screening questionnaire for essential tremor agree with the physician's examination? Neurology 1998;50:1351-1357.

20. Zheng W, Wang S, Barnes LF, Guan Y, Louis ED. Determination of harmane and harmine in human blood using reversed-phase high-performance liquid chromatography and fluorescence detection. Anal Biochem 2000;279:125-129.

21. Rajput AH, Hornykiewicz O, Deng Y, Birdi S, Miyashita H, Macaulay R. Increased noradrenaline levels in essential tremor. Neurology 2001;56(suppl 3):A302.
22. Cleeves L, Findley LJ. Propranolol and propranolol-LA in essential tremor: a double blind comparative study. J Neurol Neurosurg Psychiatry 1988;51:379-384.

23. Jefferson D, Jenner P, Marsden CD. B-Adrenoreceptor antagonists in essential tremor. J Neurol Neurosurg Psychiatry 1979;42:904-909.

24. Young RR, Growdon JH, Shahani BT. Beta-adrenergic mechanisms in action tremor. N Engl J Med 1975;293:950-953.

25. Hardebo JE, Owman C. Barrier mechanisms for neurotransmitter monamines and their precursors at the blood-brain interface. Ann Neurol 1980;8:1-11.

26. Ho BT. Monamine oxidase inhibitors. J Pharm Sci 1972;61: 821-837.

27. Ho BT, McIsaac WM, Walker KE, Estevez V. Inhibitors of monoamine oxidase. J Pharm Sci 1968;57:269-273.

28. Buckholtz N, Boggan WO. Monoamine oxidase inhibition in brain and liver produced by $\beta$-carbolines: structure-activity relationship and substrate specificity. Biochem Pharmacol 1977;26:1991-1996.

29. Neff NH, Fuentes JA. The use of selective monoamine oxidase inhibitor drugs for evaluating pharmacological and physiological mechanism. In: Monoamine oxidase and its inhibition. Ciba Foundation Symposium 39. Amsterdam: Elsevier, 1976: 163-179.

\title{
Medication adherence among HIV+ adults
}

\section{Effects of cognitive dysfunction and regimen complexity}

\author{
C.H. Hinkin, PhD; S.A. Castellon, PhD; R.S. Durvasula, PhD; D.J. Hardy, PhD; M.N. Lam, PhD; \\ K.I. Mason, PhD; D. Thrasher, PhD; M.B. Goetz, MD; and M. Stefaniak, BA
}

\begin{abstract}
Background: Although the use of highly active antiretroviral therapy in the treatment of HIV infection has led to considerable improvement in morbidity and mortality, unless patients are adherent to their drug regimen (i.e., at least 90 to $95 \%$ of doses taken), viral replication may ensue and drug-resistant strains of the virus may emerge. Methods: The authors studied the extent to which neuropsychological compromise and medication regimen complexity are predictive of poor adherence in a convenience sample of 137 HIV-infected adults. Medication adherence was tracked through the use of electronic monitoring technology (MEMS caps). Results: Two-way analysis of variance revealed that neurocognitive compromise as well as complex medication regimens were associated with significantly lower adherence rates. Cognitively compromised participants on more complex regimens had the greatest difficulty with adherence. Deficits in executive function, memory, and attention were associated with poor adherence. Logistic regression analysis demonstrated that neuropsychological compromise was associated with a 2.3 times greater risk of adherence failure. Older age ( $>50$ years) was also found to be associated with significantly better adherence. Conclusions: HIV-infected adults with significant neurocognitive compromise are at risk for poor medication adherence, particularly if they have been prescribed a complex dosing regimen. As such, simpler dosing schedules for more cognitively impaired patients might improve adherence.
\end{abstract}

NEUROLOGY 2002;59:1944-1950

The introduction of highly active antiretroviral therapy (HAART) regimens has improved the virologic, immunologic, and clinical outcomes of HIV infection. ${ }^{1-4}$ Protease inhibitors have been shown to improve neuropsychological functioning as well. ${ }^{5-8}$ These treatment advances have led to optimism among HIV-infected patients as well as among clinicians and researchers specializing in HIV/AIDS.

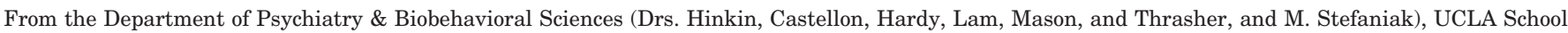
of Medicine; VA Greater Los Angeles Health Care System (Drs. Hinkin, Castellon, Lam, and Goetz); Department of Psychology (Dr. Durvasula), California State University at Los Angeles; and Department of Medicine (Dr. Goetz), UCLA School of Medicine, Los Angeles, CA.

Supported by a grant from NIMH (R01 MH58552) to C.H.H., with supplemental funds provided by NIDA.

Received June 3, 2002. Accepted in final form September 9, 2002.

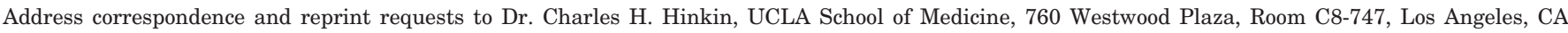
90024; e-mail: chinkin@ucla.edu

1944 Copyright () 2002 by AAN Enterprises, Inc 